\title{
Multimass thermal desorption spectroscopy as a monitoring device for chemical reaction products
}

\author{
G. Zagatta, H. Muller, N. Bowwering, and U. Heinzmann \\ Universität Bielefeld, Fakultät für Physik, 33501 Bielefeld, Germany
}

(Received 7 September 1993; accepted for publication 3 November 1993)

To observe the products of surface reaction mechanisms we combined a standard quadrupole mass spectrometer featuring high-speed scanning options with fast data acquisition and a computer-controlled sample heating system. This combination served to obtain a general view (like a "fingerprint") of the reaction occurring on a $\mathrm{Pt}(100)$ crystal upon heating the adsorbate covered surface, as well as to allow for a detailed analysis of the gas compounds leaving the surface within a single measurement. The method is denoted by multimass thermal desorption spectroscopy (MTDS). The MTDS device is capable of recording $850 \mathrm{amu} / \mathrm{s}$ providing up to 15 complete mass spectra per second of $50 \mathrm{amu}$ width while heating the surface linearly. The data contain the ion currents of both the reaction products and their fractions (cracking patterns) generated in the spectrometer. Thus it is possible to distinguish between gas species with equal mass numbers by subsequent numerical evaluation.

\section{INTRODUCTION}

Quadrupole mass analyzers (QMA) are standard devices in technical and research applications. Commercial instruments usually provide two modes of operation: (1) Tracking the partial pressure for one fixed mass number and (2) scanning a specific range of mass numbers giving the complete mass distribution for that range. Modern computer-controlled QMAs allow, as an extension of mode (1), the tracking of a set of previously chosen mass numbers in which the mass filter is tuned to the discrete mass channels within a specific time (typically some milliseconds) and then the channel is read out. The scanning mode (2) is often used for residual gas analysis to display the mass distribution on an oscilloscope or a two-axis plotter.

In experiments examining chemical reactions it is desirable to record the partial pressures of several reaction products simultaneously or in sequence at high speed, since then the system to be investigated needs to be prepared only once for a complete measurement. Especially in cases of rapid and intense reactions, fast sampling rates for each mass channel are of great importance. For this purpose, time-of-flight (TOF) mass spectrometers are well suited as recent applications show: For example, Blais et al. ${ }^{1}$ used a TOF mass spectrometer with a detection speed of $300 \mathrm{amu}$ in $12 \mu$ s to detect reaction products in explosion processes. However, TOF spectrometers are sensitive and complex devices, so in many applications researchers use conventional QMAs operating in the extended mode (1).

To carry out temperature programmed reaction (TPR) studies on the catalytic NO-CO reaction on $\mathrm{Pt}(100)$, we have developed a novel scheme for fast recording of complete mass spectra using a commercially available standard QMA. ${ }^{2}$ A configuration was set up which is capable of detecting all chemical specics involved in the reaction, including the dissociation products generated in the QMA in a single heating cycle. To this end the QMA was operated in the scanning mode (2) at a high scanning speed, the output signal was recorded by means of a computer and a fast analog-to-digital converter $(A D C)$, and the partial pressure was extracted for each mass channel from the mass spectrum by numerical methods. This method which we denote by multimass thermal desorption spectroscopy (MTDS) yields complete information on the course of the reaction and even allows determination of gas compounds when cracking patterns of the gases are considered during data evaluation.

\section{SETUP}

The TPR experiments on $\operatorname{Pt}(100)$ were part of a project to study the interaction of free oriented NO molecules with adsorbates and surfaces. The apparatus consists of a beamline to produce a supersonic state-selected molecular NO beam and an ultrahigh vacuum (UHV) chamber equipped for surface analysis. The beamline and parts of the UHV chamber are described previously. ${ }^{3,4}$

Here, we only describe the main vacuum components for the TPR measurements shown in Fig. 1. The platinum sample, cleaned by standard procedures, is exposed to both NO and CO by means of the two dosers shown. The sample holder is equipped with liquid-nitrogen cooling as well as an electron impact heating to control the crystal temperature which is monitored by a type $K$ thermocouple spot welded to the edge of the crystal. During a heating cycle the desorbing adsorbates and reaction products are detected by the QMA next to the dosers which is shielded from the residual gas by an orifice attached to the front of the analyzer head.

To fully record the gas compounds leaving the surface, the QMA control unit is set to the fastest scanning mode of $1000 \mathrm{amu} / \mathrm{s}$ (which turned out to be $850 \mathrm{amu} / \mathrm{s}$ actually) with a scanning width of $50 \mathrm{amu}$ starting at mass number 1. In this mode the control unit switches to the lowest mass number, delays for a dead time of about $7.5 \mathrm{~ms}$ to settle and stabilize the voltages applied to the mass filter, and tunes across the specified range of $50 \mathrm{amu}$ within $59 \mathrm{~ms}$, repeating the cycle continuously. The analog output signal 


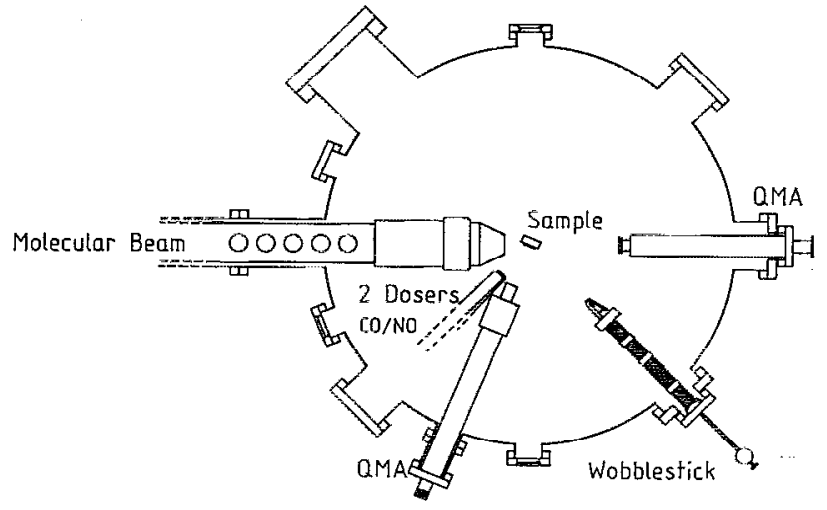

FIG. 1. Sectional drawing of the setup for TPR experiments. The platinum crystal can be exposed to both NO and CO by means of two dosers pointing to the crystal from above and below the plane shown. The QMA next to the dosers records the molecules which leave the surface during the heating cycle of the sample. The front cap of the QMA is designed to avoid detection of molecules from the residual gas.

provided by the control unit is switched to $-10 \mathrm{~V}$ during the dead time at the beginning of a spectrum and gives the ion currents on a voltage scale in the range of 0 to $+10 \mathrm{~V}$ during scanning. The output signal is proportional to the partial pressure in a selectable but fixed pressure range ( $10^{-9}$ mbar in this experiment).

The analog signal containing the complete information on the desired spectrum is connected to an 8-bit transient recorder card $^{5}$ installed on an IBM compatible 386 computer. Programmed for a conversion time of $100 \mu$ s and a data block size of 1024 bytes, the transient recorder samples the QMA signal with ten data points per amu and records a complete mass spectrum in one data block. After the recording of one block is complete, the computer reads out the data and stores them onto a RAM disk. This ensures that data transfer is finished before the next mass spectrum is transmitted by the QMA. The recorded data are saved onto the hard disk and cvaluated after the measurement is complete.

During the measurement the crystal temperature is increased linearly from -50 to $370{ }^{\circ} \mathrm{C}$ at a selectable heating rate. This process is completely software controlled with the heating power supplied by electron impact heating consisting of two programmable power supplies: a $300 \mathrm{~W}$ highvoltage supply ${ }^{6}$ and a 10 A current source. ${ }^{7}$ Both supplies are controlled by the computer via a digital-to-analog converter (DAC) with 16-bit resolution. ${ }^{8}$ The thermocouple signal is normalized to a voltage of $10 \mathrm{mV} /{ }^{\circ} \mathrm{C}$ with a special integrated circuit ${ }^{9}$ performing this conversion. Since a linear heating of the crystal means the first derivative of the temperature with respect to the time being constant and the second derivative equaling zero, two electronic differentiators are used to provide the two first derivatives as analog signals readable by an ADC. Thus the computer can access the temperature as well as its derivatives to perform a regulating algorithm for the heating supplies.

Figure 2 shows all major hardware components used for the MTDS device in a block diagram. All parts are commercial instruments except for the differentiators and

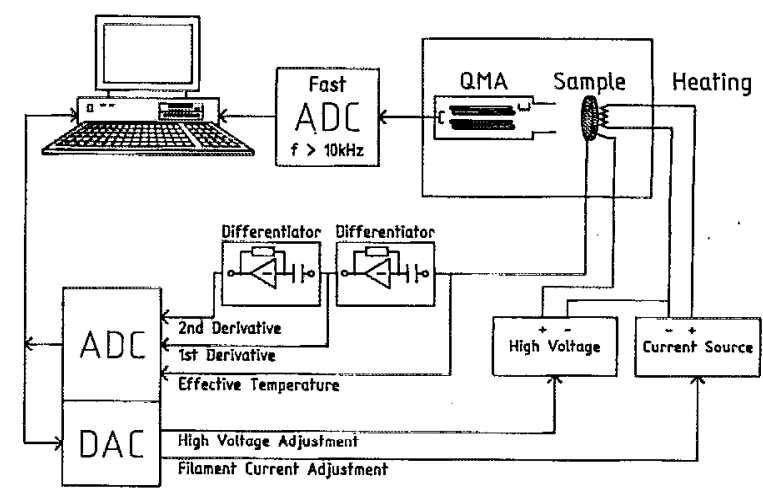

FIG. 2. Block diagram of the hardware setup for MTDS. The QMA signal is recorded by a fast ADC (transient recorder), the surface temperature is given by a type $\mathrm{K}$ thermocouple. To calculate the appropriate heating power for the crystal, the computer reads the temperature and the first two derivatives generated by two electronic differentiators. The heating power is provided by programmable power supplies controlled by the computer via a DAC.

the ADC which reads the temperature signals. They are homemade circuits following standard applications.

The apparatus described above consists of separate parts which have to be linked by appropriate software. Such a program has to control the transient recorder card and the crystal heating simultaneously. The transient recorder is essentially self-running and only needs initialization at the beginning of a run, a trigger-release command to start the measurement, and a command to transfer the data into a user RAM location. Finding the trigger, sampling the input signal and storing the data in the internal RAM is completely executed by the card itself, thus communication between the program and the transient recorder card is highly simplified.

For the heating control an algorithm had to be found to allow a well-defined startup of the power supplies and a sufficient regulation mechanism to keep the temperature's first derivative constant. In this experiment the algorithm was split into two parts. At the beginning of a heating cycle the power supplies are programmed with initial values found experimentally. After a specific settling time the computer starts to regulate the heating power with a PID characteristic. This operation scheme is rather simple but it needs carefully chosen values for the different parameters involved to perform a proper regulation. In practice one regulation step (i.e., adjusting the heating power according to the values of the current temperature and its derivatives) per measured mass spectrum is sufficient to obtain a linear temperature sweep for the crystal surface.

The flow chart of the complete program used for MTDS measurements is shown in Fig. 3. It is embedded in a larger control program providing routines for all types of measurements and calculations which are of importance in the experiment.

\section{PERFORMANCE}

According to the time settings mentioned in Sec. II; the cycle time for one mass spectrum of $50 \mathrm{amu}$ width is 


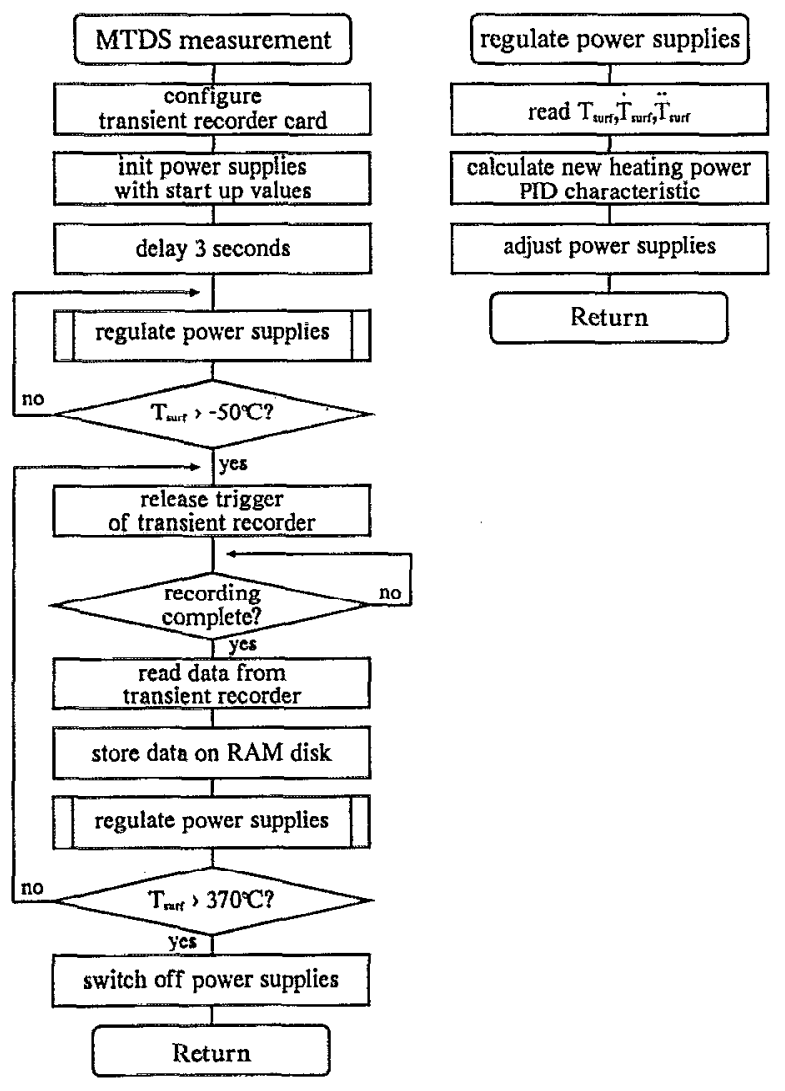

FIG. 3. Flow chart of the measurement program.

$\sim 66 \mathrm{~ms}$. This should yield up to 15 spectra per second. Since the transient recorder card can only store data in blocks of 1024 bytes (or other powers of two), it is not possible to sample exactly one spectrum; instead, the beginning to the next spectrum is additionally recorded in the same block. So only every second spectrum is complete, leading to an effective scan rate of 7.5 complete mass spectra per second for this MTDS device. A more flexible ADC could solve the problem of the lost second spectrum and allow for the maximum value of 15 spectra per second.

The high scanning speed requires fast and low-noise electronic signal amplification inside the spectrometer. If the pressure range is set to $10^{-9} \mathrm{mbar}$, the noise is typically less than $3 \%$ of the signal. In many cases this accuracy is sufficient. The internal electronic time constant which is necessary to stabilize the signal leads to a damping and broadening of the peaks in the mass spectrum. In order to extract the ion current for one mass channel as a single number, the peak is integrated within specific limits which also compensates the effects of the time constant. This can be seen from Fig. 4 where two desorption curves of $\mathrm{CO}$ from $\operatorname{Pt}(100)$ are compared. The solid line gives the MTDS signal of mass number 28 extracted from a complete spectrum, whereas the dashed line shows the corresponding signal recorded in the single-mass mode. Apart from linear scaling both curves represent original raw data without application of background correction or smoothing. The two curves are alike except for the higher noise of

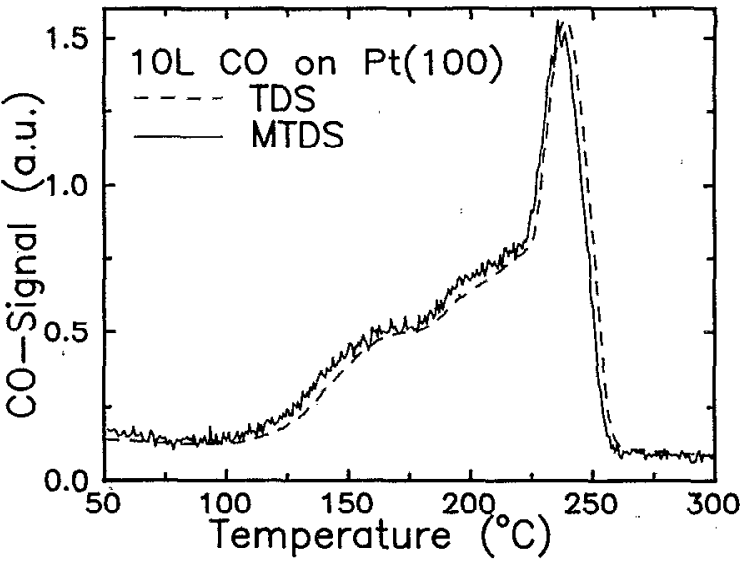

FIG. 4. Comparison between a conventional TDS (dashed line) and the corresponding mass number from an MTDS spectrum (solid line). The system $10 \mathrm{~L}$ of $\mathrm{CO}$ and $\mathrm{Pt}(100)$ was chosen. The heating rate was 4.5 $\mathrm{K} / \mathrm{s}$.

the MTDS signal and a small shift in the temperature scales.

An important limit to the performance is the fixed pressure range for one mass spectrum which must be optimized to the highest peaks. This leads to a dynamic range of about 100 thus limiting the resolution of the smaller peaks in the spectrum. The mass separation is also limited depending on the selected pressure range. For the range of $10^{-9}$ mbar the signals of two adjacent mass numbers cannot be well separated by the integration method described above.

\section{APPLICATION TO THE SYSTEM NO/CO/Pt(100)}

MTDS was used to examine the NO-CO reaction on $\operatorname{Pt}(100)$ forming mainly $\mathrm{CO}_{2}$ and $\mathrm{N}_{2}$. This reaction has been subject to several studies since 1970 (see, for example, Refs. 10-13)." The platinum crystal was precovered at $-80^{\circ} \mathrm{C}$ with both $\mathrm{CO}$ and NO sequentially and was then heated to $370^{\circ} \mathrm{C}$ at constant heating rates between 2 and 40 $\mathrm{K} / \mathrm{s}$. Several experiments were carried out where the dosage of both gases, the sequence of exposure and the heating rate were varied.

Figure 5 shows the three-dimensional representation of an MTDS spectrum for the system $10 \mathrm{~L}$ NO on $0.5 \mathrm{~L} \mathrm{CO}$ on $\mathrm{Pt}(100)$. The QMA signal is given as a function of the mass number and the temperature. A Gaussian convolution was carried out to smooth the signal. Since the measurement was performed at a constant heating rate of 4.5 $\mathrm{K} / \mathrm{s}$ the temperature axis also shows the temporal course of the reaction. At temperatures below $120^{\circ} \mathrm{C}$ NO (mass number 30 ) desorbs with a desorption curve exhibiting the well-known shapes shown in Refs. 11, 14-16. At temperatures around $120^{\circ} \mathrm{C}$ (depending on the coverage ratio of $\mathrm{NO}$ and $\mathrm{CO}$ ) the "explosive" reaction occurs with sharp desorption peaks of $\mathrm{N}_{2}$ and $\mathrm{CO}_{2}$ (mass numbers 28 and 44 ), promoted by an autocatalytic mechanism. ${ }^{12}$ The peaks at the mass numbers $12,14,16$, and 22 are due to the dissociation of the gas molecules inside the QMA and represent the ions $\mathrm{C}^{+}, \mathrm{N}^{+}, \mathrm{O}^{+}$, and $\mathrm{CO}_{2}^{++}$, respectively. 


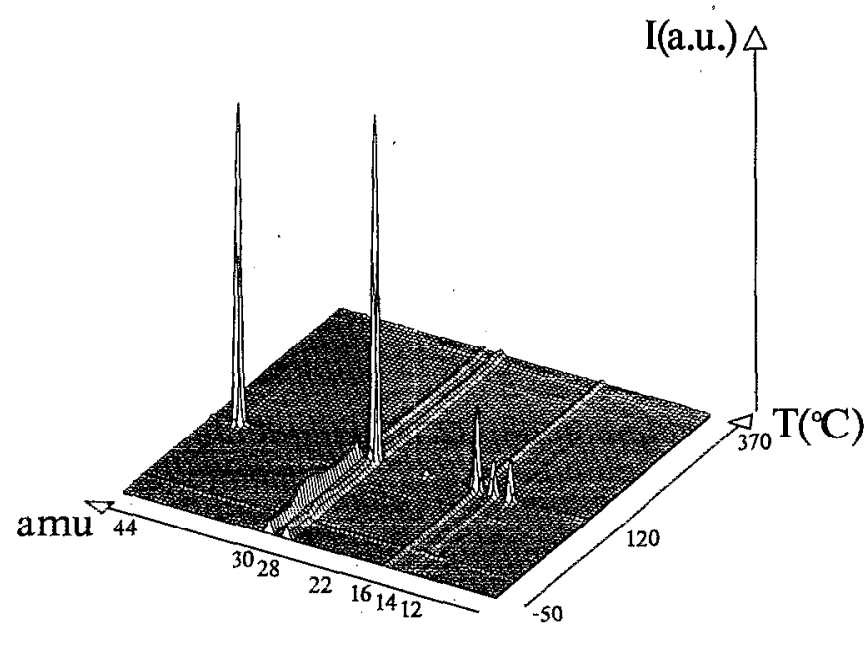

FIG. 5. MTDS spectrum of the NO-CO reaction on Pt(100) with exposures of first $0.5 \mathrm{~L}$ of $\mathrm{CO}$ and then $10 \mathrm{~L}$ of NO. The QMA signal is given as a function of mass number and temperature at a heating rate of 4.5 $\mathrm{K} / \mathrm{s}$.

After the reaction is complete there are no molecules left on the surface since the reactants were present in optimum concentration for the system shown.

Several measurements with different precoverages of $\mathrm{NO}$ and $\mathrm{CO}$ were evaluated for the signal intensities of the desorbing species. Making use of the relative sensitivities of the QMA for different gases which have been found experimentally, the true composition of the desorbing gas mixture was determined, indicating that also small amounts of $\mathrm{N}_{2} \mathrm{O}$ appear among the reaction products. This has also been found for other single-crystal surfaces of platinum. ${ }^{17,18}$

\section{v. CONCLUSION}

MTDS was designed to record surface reaction processes with a conventional QMA. In combination with a transient recorder card, electron impact heating, and two. differentiators giving the derivatives of the surface temperature with respect to the time, it forms a device which records 7.5 complete mass spectra per second, thus giving a complete indication of the course of the reaction from a single measurement. The data, if plotted in a threedimensional picture, give a "fingerprint" of the reaction and can be evaluated to gain quantitative information on the reaction products and their ratios.

Due to the fixed pressure range of the QMA, the dynamic resolution is limited to 100 . Nevertheless, the performance of the device operated with the parameters mentioned above is sufficient in terms of temperature resolution, mass resolution and signal-to-noise ratio for studies similar to the application reported here. Using a fast and flexible ADC for the recording of the QMA signal, the rate of spectra recorded can be increased to 15 per second.

\section{ACKNOWLEDGMENT}

Financial support by DFG (SFB 216) and the Commission of the European Communities is gratefully acknowledged.

${ }^{1}$ N. C. Blais, H. A. Fry, and N. R. Greiner, Rev. Sci. Instrum. 64, 174 (1993).

${ }^{2}$ Quadrupole Mass Analyzer, type Masstorr DX. Manufacturer: VG Instruments, Hastings, UK.

${ }^{3}$ G. H. Fecher, M. Volkmer, B. Pawlitzky, N. Böwering, and U. Heinzmann, Vacuum 41, 265 (1990).

${ }^{4}$ H. Müller, B. Dierks, F. Hamza, G. Zagatta, G. H. Fecher, N. Böwering, and U. Heinzmann, Surf. Sci. 269/270, 207 (1992).

${ }^{5}$ PC Transient recorder card, type TSR 12840 . Manufacturer: IMTEC GmbII, Backnang, Germany.

${ }^{6}$ High-voltage supply, type MCN 650-300. Manufacturer: FuG, Rosenheim, Germany.

${ }^{7}$ Low-voltage supply, type LNG 50-10. Manufacturer: Heinzinger, Rosenheim, Germany.

${ }^{8} \mathrm{AD} / \mathrm{DA}$ converter, type PCI 6380. Manufacturer: CIL Group, Lancing, Sussex, UK.

${ }^{9}$ Thermocouple amplifier, type AD 595. Manufacturer: Analog Devices.

${ }^{10}$ T. E. Fischer and S. R. Kelemen, J. Catal. 53, 24 (1978).

${ }^{11}$ M. W. Lesley and L. D. Schmidt, Surf. Sci. 155, 215 (1985).

${ }^{12}$ Th. Fink, J.-P. Dath, M. R. Basset, R. Imbihl, and G. Ertl, Vacuum 41, 301 (1990).

${ }^{13}$ Th. Fink, J.-P. Dath, M. R. Bassett, R. Imbihl, and G. Ertl, Surf. Sci. 245, 96 (1991).

${ }^{14}$ S. R. Kelemen, T. E. Fischer, and J. A. Schwarz, Surf. Sci. 81, 440 (1979).

${ }^{15}$ P. A. Thiel, R. J. Behm, P. R. Norton, and G. Ertl, J. Chem. Phys. 78, 7448 (1983).

${ }^{16}$ R. J. Gorte and L. D. Schmidt, Surf. Sci. 109, 367 (1981).

${ }^{17}$ R. M. Lambert and C. M. Comrie, Surf. Sci. 46, 61 (1974).

${ }^{18}$ Y. O. Park, W. F. Banholzer, and R. I. Masel, Surf. Sci. 155, 341 (1985). 